\title{
LAS FRONTERAS ENTRE DISEÑO DE MODA YTECNOLOGÍA: POSIBILIDADES PARA UNA PRODUCCIÓN SOSTENIBLE
}

\section{THE FRONTIERS BETWEEN FASHION DESIGN AND TECHNOLOGY: POSSIBILITIES FOR SUSTAINABLE PRODUCTION}

\author{
ELENIR CARMEN MORGENSTERN, Dra. | UNIVILLE \\ EFRAÍN FOGLIA, DR. | UOC \\ SILVANA SILVA REITER WITKOSKI, M.Sc. | UNIVILLE \\ LETICIA HERMES, M.SC. | UNIVILLE \\ HELENA MORGENSTERN ZAMBERLAN | UNIVILLE
}

\begin{abstract}
RESUMEN
El artículo se adentra en cuestiones relacionadas con las fronteras disciplinares del diseño. Se centra, más concretamente, en las prácticas relacionadas con los campos del diseño de la moda y la tecnología para el desarrollo de productos de moda. En este sentido, el texto destaca teorías conexas a una producción de diseño de moda sostenible evidenciando prácticas relacionadas con proyectos sociales en Brasil, de extensión universitaria, que invierten en tecnología social que, en sus métodos, procesos y materialidad, minimicen impactos ambientales. Con la intención de transponerse a la visión romántica acerca del potencial de las tecnologías para el campo de la moda, el artículo plantea cuestionamientos acerca de la contribución de los avances tecnológicos al medio ambiente, específicamente en el campo de la moda. La reflexión profundiza en la medida, en que las tecnologías sociales y los procesos tecnológicos aplicados en el desarrollo de productos de moda pueden contribuir con la sostenibilidad, minimizando los impactos ambientales. El texto en su desarrollo describe tecnologías actualmente aplicables a los productos de moda; presenta un caso ilustrativo escudriñando la intención teórica por medio de un breve historial sobre la evolución tecnológica de la pieza "camiseta" (T-shirt; semarreta); contextualiza prácticas relacionadas con la producción de moda en España/Barcelona; y, finalmente, analiza y emite posicionamientos de valor por medio de cruce entre las reflexiones teóricas y los casos prácticos.
\end{abstract}

PALABRAS LLAVE: Diseño de moda; tecnología; sostentabilidad.

\begin{abstract}
The article delves into issues related to the disciplinary boundaries of design. It focuses more specifically on practices related to the fields of fashion design and technology for the development of fashion products. In this sense, the text highlights theories related to a production of sustainable fashion design evidencing practices related to social projects in Brazil, university extension, that invest in social technology through methods, processes and materials that minimize environmental impacts. The article raises questions about the contribution of technological advances to the environment, specifically in the field of fashion. The reflection questions the extent to which social technologies and technological processes applied to the development of fashion products can contribute to sustainability, minimizing environmental impacts. The text, in its development, describes technologies currently applied to fashion products; presents an illustrative case examining the theoretical intention by means of a brief history about the technological evolution of the piece "T-shirt" (semarreta); contextualizes practices related to fashion production in Spain / Barcelona; and, finally, analyzes and emits positions through the intersection between theoretical reflections and practical cases.
\end{abstract}

KEY WORDS: Fashion design; technology; sustainability. 


\section{INTRODUCCIÓN}

Los datos y reflexiones presentados son el resultado de una pasantía posdoctoral (por Elenir Morgenstern bajo la supervisión del profesor Dr. Efraín Foglia) en la Universidad de Barcelona (UB), con investigación incluida en la línea de investigación Imagen y diseño, vinculada al programa de posgrado Estudios Avanzados en Producción Artística.

El artículo se centra en las prácticas relacionadas con los campos del diseño de la moda y la tecnología para el desarrollo de productos de moda sostenible, adentrando en cuestiones relacionadas con las fronteras disciplinares del diseño. Por tecnología se entiende, en este texto, el conocimiento técnico científico y sus aplicaciones a un campo particular. El concepto de tecnología, en el campo específico de la moda, es ampliamente abordado por Fletcher e Grose (2011) tratando de estudios sistemáticos acerca de técnicas, procesos, métodos, medios e instrumentos de aplicados específicamente a los procesos de moda.

En este sentido, el artículo destaca teorías conexas a una producción de diseño de moda sostenible evidenciando prácticas relacionadas con proyectos sociales, de extensión universitaria, que invierten en tecnología social que, por medio de manufacturas, en sus métodos, procesos y materialidad, minimicen impactos ambientales.

Es necesario aclarar que, con la expresión diseño para la sostenibilidad entendemos el acto de proyectar productos, servicios y sistemas con un bajo impacto ambiental y una alta calidad social; cuando hablamos de sostenibilidad ambiental relacionada al desarrollo de productos, nos referimos a los criterios, a los métodos ya las inversiones del mercado (VEZZOLI, 2008).

A partir del cruce entre los enfoques teóricos que anuncian tecnologías de última generación, aplicables en el desarrollo de productos de moda, y las prácticas analizadas junto a algunos proyectos sociales, de extensión universitaria, impulsados por los saberes del diseño de moda, el artículo plantea cuestionamientos acerca de la efectiva contribución de los avances tecnológicos al medio ambiente, específicamente en el campo de la moda. La reflexión presentada, con el fin de transponerse a la visión romántica acerca del potencial de las tecnologías para el campo de la moda, indaga de hecho, y en qué medida, las tecnologías sociales y los procesos tecnológicos aplicados en el desarrollo de artefactos de moda pueden contribuir con la sostenibilidad, minimizando los impactos ambientales. La intención del texto, en su conjunto, analiza el diseño de moda y las perspectivas de sostenibilidad; describe tecnologías actualmente aplicables a los artefactos de moda; presenta un caso ilustrativo escudriñando la intención teórica por medio de un breve historial sobre la evolución tecnológica de la pieza "camiseta" (T-shirt; semarreta); contextualiza prácticas de tecnología social promovidas por proyectos de extensión universitaria en Brasil; $y$, finalmente, analiza y emite posicionamientos de valor por medio de cruce entre las reflexiones teóricas y los casos prácticos.

Al lector neófito, la redacción puede parecer multifocal; sin embargo, hay que evidenciar que la propuesta es intencional: la perspectiva ofrecida trata el campo de la moda como un sistema simbólico, interrelacionado con otros campos que, en su conjunto, constituyen un sistema mayor. Por esto motivo, estando convencidos de que no se llega a la comprensión analizando separadamente las partes (conocimiento disciplinario de la Modernidad), se propone el análisis del diseño de moda entrelazado en una tela (CAPRA, 2013), a otros campos, considerando en medio de los campos, las prácticas de los agentes. Así, en una perspectiva antropológica, se intenta un posicionamiento teórico a partir de las prácticas de los agentes y no meramente soportados por enfoques teóricos.

\section{EL CAMPO DE LA MODA}

El campo de la moda (diseñadores, productores, mercado...), en gran medida, se ha inclinado hacia una producción insostenible. La industria de la moda viene destacándose por el fast fashion (moda rápida), en producciones tendenciosas y de baja calidad en lo relacionado a materiales y procesos. Los artefactos, producidos y comercializados a gran escala, con bajos precios, estimulan el descarte y el ciclo vicioso en el sistema que sostiene su mercado.

Otro aspecto relevante está relacionado con el estímulo del consumo por medio de la producción de "objetos del deseo" (FORTY, 2007). La diversidad de los diseños de objetos, como bien destacó el historiador del diseño Adrian Forty, lleva a creer que siempre necesitamos algo más, pues el detalle, la forma, el color de un artefacto supera a su antecesor moviendo nuevas necesidades. Las tendencias, efímeras por naturaleza, hacen obsoletos incluso los productos que aún están en los estantes. Y, además, el aura trascendental de las marcas, destacadas en medio de cada sociedad, denotan el valor simbólico que va siendo instituido en la propia cultura (ver BOURDIEU, 2006).

Los enfoques teóricos, nacidos en buena parte en contextos académicos, han alertado sobre problemas generados por las prácticas productivas y comerciales de la industria de la moda. Surgen teorías relacionadas con la eco-moda, moda consciente, sostenibilidad para la moda, slow fashion, movimiento maker, entre otros. 
El movimiento Eco-Moda se desarrolla pautado en el respeto al medio ambiente, ese perfil de producto/marca de moda ha conquistados adeptos principalmente en Europa. Bajo impacto ambiental, fibras naturales y orgánicas, teñidos ecológicos, reciclaje de objetos y prendas de vestir son la base en la que se sustentan dichas prácticas proyectuales (SCHULTE; LOPES, 2008).

El "Slow Fashion" se configura en movimiento inspirado en "Slow Food", propone la producción de ropas para ser usadas, mantenidas y cuidadas. Se trata de piezas de ropa transsazonales, es una propuesta contraria al Fast Fashion, rompiendo con las prácticas actuales de la industria de la moda, orientadas siempre al crecimiento (FLETCHER; GROSE, 2011).

Moda Consciente y sostenibilidad para la moda encajan en los principios definidos por la "ecomoda".

El movimiento "maker" propone el uso de herramientas digitales o manuales, así como el conocimiento técnico democratizado, para que comunidades, en redes globales, puedan producir por sí mismas los artefactos. Un autor importante, que ayudó en la eclosión del Movimiento Maker fue Chris Anderson, que definió a los fabricantes de una forma más estructurada: los fabricantes están llevando el movimiento "hágalo usted mismo" a las comunidades globales, con tres características principales: uso de herramientas digitales para diseñar y modelar proyectos; cultura de compartir proyectos de diseño y de colaborar con otras comunidades en red; el uso de bases de datos de diseño común que democratizan el acceso a los desarrollos de los proyectos (ANDERSON apud MANICHINELLI, 2017).

Por otro lado, la concepción contemporánea acerca delo que entendemos por conocimiento demuestra la evanescencia entre las fronteras de distintos campos, como por ejemplo la moda y la tecnología. El acceso casi ilimitado a los nuevos saberes producidos permite, en el mercado de la moda, una producción híbrida (CANCLINI, 2003) con lo que no solo vemos ropa o accesorios, o ni identificamos meramente tejido o materiales tecnológicos; vemos algo nuevo, una nueva cosa, que evidencia el cruce entre los saberes de ambos campos.

\section{EL CAMPO DE LA TECNOLOGÍA Y POSIBILI- DADES EXTENDIDAS AL DISEÑO DE LA MODA}

La aplicación de la tecnología en los procesos en diseño de moda posibilita el desarrollo de tejidos inteligentes y anuncia la institución de nuevos hábitos (sociales) relacionados con cuerpo y la vestimenta. Estas prácticas se han denominado "wearable" o "tecnologías usables híbridos que surgen de la unión de las dos áreas y acrecientan otras posibilidades para el diseño de la moda.
Empresas y escuelas desdoblan investigaciones posibilitadas por el cruce de los conceptos de moda y los procesos tecnológicos actuales.

La empresa japonesa Riken (2017) presenta estudios desarrollados en conjunto con la Universidad de Tokio en la creación de una célula solar lavable capaz de suministrar energía para equipos electrónicos. De acuerdo con sus productores, el dispositivo se mostró eficiente cuando se probó debajo del agua o bajo compresión y distensión. Esta tecnología apunta la posibilidad para el desarrollo de células solares portátiles que puedan abastecer dispositivos acoplados a la vestimenta.

El sitio del grupo Rhodia (2017) divulga la creación de un hilo, denominado Emana. El hilo, constituido por minerales bioactivos, posibilita absorber el calor del cuerpo y transmitir a la misma onda de infrarrojo que auxilia en la circulación sanguínea y en la temperatura corporal.

Recientemente, la compañía Sasawashi (2017) anunció el desarrollo de un tejido a partir de la técnica de producción del papel washi. El material, que se define sostenible es fabricado en conjunto con fibras de la planta kumazaca, que posee propiedades antibacterianas y desodorantes. Además, el papel washi posee ligereza, no hay pelusas, propicia la protección UV y demuestra alta durabilidad, manteniendo sus propiedades después de varios lavados. De acuerdo con el diario The Guardian (2017), la startup Qmilk creó, a partir de leche agria, una fibra sostenible que tras una mezcla especial producida en laboratorio se transforma en líneas y consecuentemente en tejido. La fibra, generada a partir de la leche, presenta un aspecto similar al de la seda, pero con el diferencial de no utilizar pesticidas para ser constituido, lo que lo hace $100 \%$ natural. De acuerdo con la citada fuente, este tejido también presenta una función antibacteriana y se muestra eficiente en circunstancias de regulación de la temperatura corporal.

Aparte de los desdoblamientos mercadológicos, hay que destacar las investigaciones presentadas en un importante congreso brasileño "Il Congresso Brasileiro Design e Materiais". Investigaciones vinculadas al campo de la moda vienen destacando ensayos que aplican las nuevas posibilidades tecnológicas. Entre las investigaciones socializadas, destacamos el experimento relacionado con procesos creativos de estampación a partir del cultivo de hongos y bacterias ("BioStudio: seres vivos, tejidos e innovación" presentado por Breno Tenório Ramalho de Abreu a congreso Diseño y Materiales, 2017) e investigaciones desarrolladas por la Universidad de la Región de Joinville UNIVILLE (SETE; MORGENSTERN, 2017), que invierten en la proposición de un nuevo hilo a partir de la mezcla entre 
fibra de la "paina" y fibra del "pseudo-caule" del plátano ("Fibras Naturales en la producción de hilos textiles: mejoras en el estudio del uso de la fibra de Paineira y aplicaciones en artefactos de Diseño de Moda"). Los experimentos que invierte en la transformación textil por medio de la electrónica y la nanotecnología (BERGMANN; MAGALHÃES, 2017). Aun así, algunas publicaciones recientes, en formato libro, destacan el interés por las discusiones que relacionan moda y tecnología, como "Moda, globalización y nuevas tecnologías", de Avelar (2009).

\section{MODA, TECNOLOGÍA Y SOSTENIBILIDAD, LAS DOS CARAS DE LA MONEDA}

Anteriormente hemos discutido sobre la producción de artefactos de moda. La intención es averiguar si la inclusión de nuevas tecnologías en las producciones de grupos de artesanos, pueden realizarse de forma sostenible. Entendemos de antemano, que la mera aplicación de tecnologías puede, al contrario de lo esperado, generar nuevos problemas al medio ambiente $y$, por consiguiente, a afectar a las sociedades en su conjunto. Pensamos que los intercambios comerciales deben ser consecuencia de un proceso de reflexión en sintonía con el momento actual de nuestra sociedad, entendiendo que ese debate, debe ser promovido en el escenario de una sociedad que pretende ser participativa y cuestionadora de los sistemas provenientes del capitalismo voraz (FOGLIA, 2017). Creemos que la evolución tecnológica no puede basarse en la referencia de los beneficios de vender tecnologías, si no que se hace necesario averiguar concretamente cuáles son los beneficios sociales que obtenemos al utilizarla.

Nuestro interés en este artículo es desplegar, más adelante, las prácticas de proyectos sociales, cuya realización está conectada a una tecnología social y, en las cuales, el apoyo en tecnologías del diseño de moda (materiales, métodos y procesos) son aplicados con el fin de efectuar una producción sostenible que considere el medio ambiente conectada al paradigma ecológico de la contemporaneidad (CAPRA, 2013). Sin embargo, nos parece pertinente, antes de entrar en el ámbito de estos proyectos, presentar brevemente un estudio de un caso que evalúa la relación entre producción tecnológica e impactos ambientales. Así, presentamos una breve historia de la camiseta (T-shirt; semarreta), esta labor la llevaremos a cabo apoyados en una fuente histórica específica: Boucher (2010).

Los orígenes de la camiseta son antiguos, los romanos de la antigüedad ya usaban una túnica doble, llamada suéter, que es el ancestro de nuestras camisetas. Era siempre blanca, hecha casi siempre de lino. Se utilizaba debajo de la única para proteger de la transpiración. En el siglo IV, en Constantinopla, seguía utilizándose bajo la ropa. Los tejidos de las piezas superiores eran muy ricos, bordados con oro, plata y piedras preciosas, y por eso no eran lavados. El suéter se utilizaba debajo de esas piezas nobles para evitar que se ensuciara. En el siglo XIV, Miguel Ángel, en su estatua "El esclavo moribundo", ya retrata a un hombre vestido exclusivamente con una prenda muy diferente de las usadas en la época: una camiseta sin mangas. Miguel Ángel anunciaba una moda que más tarde sería de referencia (lo que hoy los diseñadores llamarían "el buen diseño"). En el siglo XIX, la ropa de los niños empieza a ser más infantil, en lugar de ser reproducciones de las de los adultos en miniatura. "Camisia" era la única vestimenta hasta los 5 o 6 años, usada también para bautizar a los niños. A principios del siglo XX la camiseta, todavía era usada como ropa de interior, para proteger a los hombres de la transpiración y del frío. Para no rasgar las camisas, los trabajadores usaban sólo la camiseta para trabajar. A partir de la 1a Guerra Mundial, soldados europeos usaron, bajo los uniformes, cómodas camisetas de algodón. Los estadounidenses, muriendo de calor en sus uniformes de lana, adoraban la novedad de las camisetas y la llevan a Estados Unidos. El diseño en formato de T Ileva la pieza a ser conocida como camiseta, en inglés. En la $2^{\text {a }}$ Guerra Mundial - La camiseta es pieza clave en el uniforme de la Armada y del Ejército Americano siendo todavía considerada ropa de interior, pero el público se acostumbra a ver en las revistas fotos de los soldados con camiseta, sin camisa por encima, al hacer trabajos pesados o en lugares calientes. En 1948 el candidato a la presidencia de Estados Unidos, Thomas E. Dewey, hace una de las primeras camisetas de propaganda de la historia, con las palabras "Dew it for Dewey". A partir de ahí, en la década de 1950, celebridades pasan a aparecer en películas vistiendo camisetas sin camisa por encima. En los años 60, los movimientos antiguerra y a favor de la libertad, comienzan a usar la camiseta con colores psicodélicos-hippies que ya cuentan con mensajes pacifistas impresos, en la línea de "Hacer Amor, No Hacer Guerra". En esa época, las mujeres también pasan a usar la pieza, que se vuelve unisex. En los años 70 las camisetas se utilizan tanto como medio de expresión de los anhelos de la juventud como como soporte para propaganda, cargando símbolos de marcas de refrigerante. Años 80, década de los yuppies, jóvenes ligados al consumismo y al individualismo, la moda pasa a ser ostentación de dinero y poder, y la camiseta empieza a traer las marcas de las marcas en gran tamaño. En los años 90, la falta de ideología de los jóvenes de la década aparece 
en las ropas largas y largas de los grunges. La camiseta es usada por cualquier segmento de la sociedad, sin ningún compromiso político, sin ideologías o delimitación de edad. En los años 2000, la camiseta continúa siendo una ropa democrática y sirviendo a todos los gustos, desde las campañas políticas, pasando por la promoción de películas, entre otros usos.

Por fin, hasta el comienzo del siglo XX, la mayor pretensión de una camiseta era proteger a los hombres de incomodidades como la transpiración. Si se exhibían, era en el cuerpo de trabajadores: verduleros, jornaleros y obreros en general. La conexión con la idea de libertad y confort estuvo siempre presente en la historia de la camiseta. En 1934, durante sus vacaciones en la Riviera Francesa, Coco Chanel apareció vistiendo un pantalón masculino y una camiseta de marinero, hecha de un tejido tan "inadecuado, miserable, frágil y bueno sólo para ropa de interior" (como escribió Edmond Charles-Roux en "Le Temps Chanel -" Los Tiempos de Chanel", sin traducción en portugués), que parecía impensable que estuviera siendo exhibida por una de las estilistas más exitosas de todos los tiempos. El mensaje era claro: el confort estaba de moda. Y ninguna otra pieza podría ser una mejor aliada que la ropa suave de algodón. En la secuencia histórica, la camiseta asumió un potencial de marketing siendo que muchas empresas pasaron a colocar estampas en las camisetas (https://super.abril.com.br).

De esta manera, en la historia de la camiseta, la gran revolución de los años 90 podía ser vista en su forma y tejidos. Telas hechas de bolsas de supermercados, de tejidos sintéticos; con cortes en $\mathrm{V}$, sin mangas, con una sola manga, con una amplia gama de colores. El formato en $T$ se estaba transformando en todas las formas posibles.

Actualmente, la camiseta se presta tanto para lides domésticas como para eventos sociales de lujo. La innovación tecnológica en su historia se nota más recientemente (a partir de la década de 2000) en la aplicación de tejidos compuestos por hilos de orígenes y composiciones diversas y por medio de procesos variados.

Tenemos hoy camisetas en hilos de seda, nylon, algodón y sintéticos de las más osadas y efímeras conjugaciones. Nos proponemos, a continuación, analizar algunos de los resultados de las tecnologías aplicadas vislumbrando los efectos e impactos ambientales provenientes de las innovaciones:

- Fibra de algodón: El algodón es una de las fibras más utilizadas en el área textil. Se trata de una fibra natural, considerada mejor que otros productos sintéticos, pero que produce un impacto ambiental significativo. "El algodón es una cultura comercial de gran importancia económica, siendo cultivado en más de 70 países. La producción mundial de la fibra es superior a 19 millones de toneladas" (SOUZA, 2000, P.88). Los avances tecnológicos para la siembra y el aprovechamiento de la fibra trajeron beneficios económicos y una reducción del tiempo en los procesos, sin embargo, impactaron negativamente en el terreno. Según Capra (2013, P.195), la utilización de máquinas en el cultivo del algodón fue responsable del aumento significativo de la producción, no obstante, "el uso masivo de fertilizantes y pesticidas químicos cambió todo el modo de hacer agricultura". De acuerdo con el mencionado teórico, a medida que las mismas especies fueron siendo plantadas año tras año y fertilizadas sintéticamente, el equilibrio de los procesos ecológicos del terreno se rompió; la cantidad de materia orgánica disminuyó y, con ella, la capacidad de este de retener la humedad. Capra alerta que los cambios en la textura de la tierra acarrearon consecuencias nocivas interrelacionadas: pérdida de humus, suelo seco y estéril, erosión por el viento y el agua, etc. Igualmente, la aplicación de productos químicos y pesticidas, además de perjudicar el terreno, es también prejudicial para la salud de los agricultores. El uso de combustible en las máquinas para la cosecha del algodón, pulverización, desmotado, envasado y para el transporte de las fibras, causa liberación de dióxido de carbono en la atmósfera, contaminando el medio ambiente, sin contar que la fibra pierde calidad en muchos de estos procesos realizados con máquina (CAPRA, 2013, p. 195). Vemos que tiene en cuenta también que, en los procesos de transformación y acabado, se aplican colorantes a gran escala para el teñido y estampado - para una camiseta de 200 gramos se utilizan alrededor de 16 a 20 litros de agua, además de productos químicos para el blanqueamiento (BERLIM, 2012).

Por último, la industria textil es responsable de generar muchos residuos en los procesos de tejer y cortar. En la página web de Dalila Textil, empresa con sede en Jaraguá do Sul, se informa que las pérdidas en los procesos representan entre el 15 y el $30 \%$ de todo lo que se consume. No se tienen datos estadísticos de los residuos generados por la sociedad, una buena parte se dona y otra va a la basura. Estas piezas de ropa, con composición 100\% algodón, son biodegradables y tardan alrededor de 1 año en descomponerse en la naturaleza;

- Algodón Orgánico: La producción de algodón orgánico, aún pequeña, viene creciendo lentamente y promoviendo cambios en el mercado. Todo algodón para ser comercializado como orgánico necesita certificación. En Brasil, esta certificación es hecha por una agencia, la Federación Internacional de Movimientos de Agricultura 
Orgánica (IFOAM). Todo el proceso es inspeccionado y garantiza que el algodón orgánico se produzca dentro de un conjunto mínimo de normas. El cultivo del algodón orgánico, de acuerdo con Souza (2000), prioriza la rotación de los cultivos para disminuir el desgaste del terreno; utiliza compuesto y abono orgánico; la siembra es hecha manual y mecánicamente; asimismo, implica la utilización de insectos benéficos y el uso de trampas para el control de plagas en lugar de pesticidas.

El proceso de hilado en el algodón convencional y orgánico es igual, pero el algodón orgánico pasa por certificación. En el proceso de transformación de los tejidos orgánicos existen diferencias. Souza (2000) explica que "los colorantes utilizados son de bajo impacto ambiental y las cantidades de agua y de energía utilizadas en el proceso son más reducidas". Uno de los problemas planteados es el coste de implementar medios menos contaminantes en el tinte, ya que el valor de estos equipos es muy elevado. Los residuos textiles de ambas materias primas (orgánica y convencional), son iguales. Las dos fibras son biodegradables y tienen una descomposición más rápida en la naturaleza. El cultivo del algodón orgánico trata mejor el medio ambiente ya que el avance tecnológico incide positivamente promoviendo un cultivo sostenible. La certificación del material es un factor preponderante para la garantía en los procesos y la fiabilidad para los compradores.

- Fibra del Poliéster: Bezerra (2017) explica que es una fibra química hecha de polímeros sintéticos, provenientes del petróleo. Estas fibras fueron creadas para sustituir las naturales, siendo más fuertes y resistentes a temperaturas más elevadas y generando mayor productividad. Normalmente pasan por el proceso de extrusión, para luego transformación en hilo, que ocurre por monofilamento (un solo filamento continuo), multifilamento (la unión de dos o más filamentos) y fibra cortada (cuando el filamento es cortado en tamaños determinados, pudiendo ser mezclado con las fibras del algodón, viscosa, nylon, lino, etc., formando la mezcla). En cuanto a los beneficios generados por la tecnología aplicada a este hilado, se evidencia que la camiseta hecha de poliéster posee alta resistencia a la humedad, se arruga con menos frecuencia, seca rápidamente y no se desvanece, además de ser más ligera que el algodón. El aspecto negativo, en el uso de la camiseta de esta fibra es que, por ser sintética, retiene el calor del cuerpo, generando sudor y mal olor. Igualmente, está el factor estético negativo, pues puede generar "bolitas" al frotarse. La tecnología aplicada a la fibra, al provenir de combustible fósil no renovable, provoca aspectos negativos al medio ambiente. Cuando tiramos una camiseta con esta composición, se mantiene mucho tiempo en la naturaleza. La fabricación de poliéster utiliza grandes cantidades de agua para su enfriamiento, junto con una gran cantidad de químicos nocivos, como lubricantes, que pueden convertirse en fuentes de contaminación si no se toman los cuidados debidos. El proceso de producción también utiliza grandes cantidades de energía. El poliéster no es biodegradable, pudiendo tardar hasta 400 años en descomponerse en la naturaleza. "Otro problema ambiental que envuelve al poliéster es la contaminación por micro plásticos, que son partículas de plástico de tamaño entre $1 \mathrm{~mm}$ y $5 \mathrm{~mm}$. Estas micro partículas de plástico se desprenden de los tejidos en los lavados y van a los ríos y mares, causando la contaminación de las playas y perjudicando a los animales marinos. Los experimentos de muestreo de aguas residuales de lavadoras domésticas demostraron que una sola pieza de prendas puede producir 1900 fibras por lavado, según Browne et al. (2011). Esto sugiere que una gran proporción de fibras micro plásticas encontradas en el ambiente marino proviene, como consecuencia, de lavado de ropa. De acuerdo con la mencionada fuente, por tratarse de una fibra sintética, los tejidos $100 \%$ poliéster pueden ser reciclados, volviéndose a convertir en fibra y generar nuevos productos. Hoy en día ya existen empresas que transforman botellas PET en tejido $100 \%$ poliéster o las mezclan con otra fibra, como la de algodón. En este caso, este último producto, ya no puede reciclarse más, debido a la dificultad de separar las fibras. Desafortunadamente, el proceso de reciclaje de botellas PET encarece los productos debido al precio de la maquinaria utilizada, sin contar que la calidad de esta segunda fibra no es igual a la de la primera fabricación.

- Mezcla de fibras: La más famosa mezcla de fibras para la producción de camisetas es la "mezcla íntima" de algodón con poliéster, normalmente $67 \%$ poliéster y $33 \%$ algodón o $50 \%$ poliéster y $50 \%$ algodón. Algunas empresas también optan por la mezcla con la fibra de la viscosa - algodón y viscosa o aún algodón, poliéster y viscosa. Mezcla íntima es un proceso realizado en el hilado, en el cual la materia prima tratada (fibras) es transformada en hilo. Estas fibras se cortan en tamaños similares - fibra del poliéster y del algodón - y son retorcidos, formando un hilo simple. Este hilo puede volver a ser retorcido con otro hilo sencillo para formar un hilo retorcido de dos cables y éste puede sufrir el mismo proceso con el fin de obtener un hilo más grueso y/o patrón diferenciado según el sector de desarrollo y aceptación del mercado. El problema con esta mezcla de fibras para la confección de camisetas es que las fibras ya no pueden separarse, imposibilitando el proceso de reciclaje, en el cual ambas vuelven a convertirse en materia prima. 
- Fibra de viscosas: Fibracel (2000) nos dice que los hilos y fibras de (raiom) viscosa, fibras químicas artificiales, son producidas por medio de la extracción de la celulosa encontrada principalmente en la madera de árboles de rápido crecimiento, pobres en residuos y fácilmente transformadas en pulpa o en el lenter de la semilla del algodón. Se forma una pasta celulósica que por extrusión en hileras y con el contacto de otras soluciones se hace la fibra. El problema en la utilización de esta fibra, según el mismo manual, es que cerca del $30 \%$ de la viscosa utilizada en la confección de ropas procede de árboles de bosques nativos y amenazados de extinción, incluyendo la Amazonia. Estamos hablando de la deforestación, entonces, aunque la viscosa provenga de fuentes renovables, la extracción y el consumo de materia prima ocurren en pasos mucho más rápidos que la capacidad de renovación de esos bosques.

En cuanto a las decisiones de los agentes consumidores en las elecciones de los productos, como existen pocos órganos que fiscalizan y certifiquen los materiales y procesos, y los pocos existentes son muy caros, todo sucede a través de la confianza. En el caso de las empresas de servicios públicos, se debe tener en cuenta que, en el caso de las empresas, su palabra es, por regla general, evaluada de modo indirecto por el comprador y representa la confianza basada en la evidencia externa y en el grado de honestidad, competencia y determinación de la calidad del producto ofertado (SOUZA, 2000, P.87).

\section{PRÁCTICAS DE PRODUCCIÓN ASOCIAN- DO DISEÑO DE MODA Y TECNOLOGÍA}

A partir de las reflexiones desarrolladas más arriba, acerca de la relación entre las tecnologías de punta y efectividad de una producción sostenible, proponemos analizar, en consecuencia, el caso específico de proyectos sociales de producción artesanal, desarrollados en el sur de Brasil. En primer lugar, es necesario mencionar que, a partir de investigaciones realizadas en 2015 y 2016 (HERMES; MORGENSTERN, 2017), se evidenció que en la región Sur de Brasil se crean proyectos sociales, vinculados a la "extensión universitaria", que, apoyados en los conocimientos del diseño, capacitan y promueven la creación manual de productos para su comercialización y generación de ingresos:

a) SempreViva: es un proyecto que se desarrolla por medio de una asociación entre la UNIVILLE - Universidad de la Región de Joinville y la SAS Secretaría de Asistencia Social de Joinville, apoyado por empresas asignadas en la región catarinense. Intenta formar entre 25 y 30 mujeres anualmente, con edades entre 18 y 55 años, a partir de prácticas y teorías pautadas en los saberes del diseño, buscando generación de trabajo y renta. Se mantiene activo desde 2007;

b) Recosturas de la Moda: con apoyo de la Feevale (universidad de la ciudad de Novo Hamburgo) propone capacitar a mujeres de barrios en situación de riesgo, vulnerabilidad social, con edades entre 20 y 70 años. Intenta unir prácticas de artesanía y conocimientos del diseño de moda, para capacitación para la creación de productos ambientalmente conscientes. El objetivo de este trabajo es estimular la socialización y la emancipación productiva de dichos agentes, teniendo en cuenta la formación cualificada, el ejercicio de la ciudadanía, la actualización para el trabajo y la generación de ingresos. Fundado en 2008, se mantiene activo, a pesar de algunas modificaciones;

c) Asociación Damas \& Tramas: resultado de una asociación entre diseñadores y artesanos de la ciudad de Caxias do Sul, con apoyo de la UCS - Universidad de Caxias do Sul. Tiene como objetivo incluir la artesanía como valor simbólico aliado a productos de moda y el diseño. Se desarrolla con éxito desde 2010, empezó como proyecto de extensión de ProModa y se ha convertido en Asociación Damas \& Tramas. Busca en sus acciones, como asociación, formalizar y poner en valor el mercado de actuación de artesanos;

d) Mujeres del Barrio: contempla el grupo de proyectos de extensión de la Univali - Universidad del Valle de Itajaí, campus de Balneário Camboriú. Tiene como objetivo la formación a partir de la teoría y las prácticas provenientes del diseño de moda y de la confección. El público objetivo de esa formación congrega a mujeres jóvenes, adultas y ancianas, buscando la orientación hacia el mercado de trabajo relacionado con la moda. En desarrollo desde 2007, el proyecto se basa en el concepto de que la formación puede ser el engranaje del cambio social y de la generación de renta y trabajo. Nuestra aportación, para el desarrollo del artículo y cruce entre tecnologías y diseño de moda, se posicionará en los proyectos desarrollados por UNIVILLE, en la ciudad de Joinville. Estos proyectos, fundados en el ámbito de la tecnología social, buscan innovación a través de prácticas hibridas entre diseño y artesanía. Las prácticas de tales grupos, extensivas a toda la sociedad, se desdoblan en los siguientes aspectos: todos los proyectos sociales están conectados a un Programa denominado ModaViva; el programa, a su vez, está vinculado al área de investigación y desarrollo de la universidad comunitaria de la Región de Joinville-Sc. Este proyecto 
de investigación, que agrupa todas las investigaciones relacionadas con los proyectos de desarrollo, se denomina SIMBOL, el cual está registrado en el Grupo de
Investigación 'Diseño, cultura y sociedad' registrado en el CNPQ (Brasil). El programa ModaViva, según la figura 1, reúne los siguientes proyectos relacionados:

\section{ModaVivas}

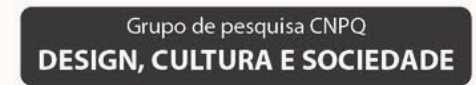

Projeto de Pesquisa PPGDesign SIMBOL

Laboratório de estudos

ModaVivas

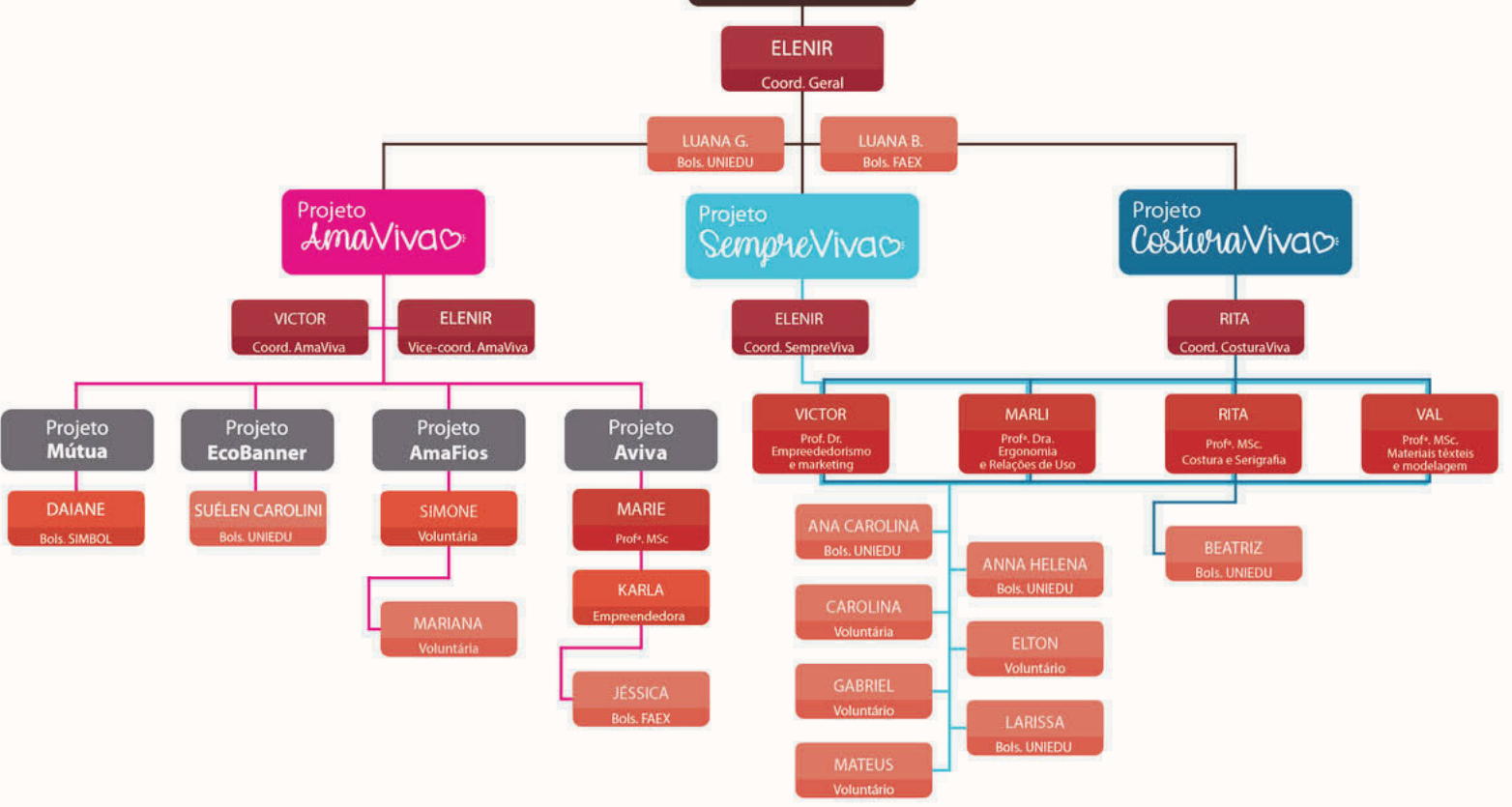

Figura 01 - Organograma Proyectos AMAVIVA/SEMPREVIVA.

Fuente: Autores.

Pasaremos al caso específico del proyecto AmaViva. El proyecto AmaViva congrega artesanas, provenientes de otros proyectos desarrollados por la misma universidad, que capacitan para el trabajo y la rentabilidad, con miras a la producción y comercialización de productos. El público objetivo está, por consiguiente, constituido por artesanas provenientes de los proyectos desarrollados en un histórico de diez años por la propia universidad en asociación con la Secretaría de Asistencia Social de Joinville. Cada año un número considerable de artesanas es formado por los referidos proyectos $y$, al término de la formación, algunas artesanas montan su propio negocio o se integran en el mercado de trabajo. Sin embargo, muchas sienten necesidad de proseguir sus actividades asociadas a un grupo. Así, el proyecto AmaViva posibilita esa oportunidad de seguir sus producciones de manera asociada y manteniendo la orientación de los profesores vinculados a los
Cursos de Diseño y Administración de UNIVILLE. El grupo está constituido por mujeres de familias con rentas hasta la mitad del salario mínimo per cápita, sin cualificación profesional y con baja escolaridad. El proyecto (fruto de asociación entre la UNIVILLE y la Secretaría de Asistencia Social) invierte en la cualificación profesional permanente de las artesanas, objetivando inserción social de las integrantes (por la generación de ocupaciones productivas y de renta). Los materiales utilizados, en buena parte, provienen de las donaciones de las asociaciones establecidas con las empresas.

El proyecto AmaViva, así como los demás proyectos de extensión está vinculado al Programa ModaViva y el referido programa, a su vez, integra las acciones del Grupo de investigación 'Diseño Cultura y Sociedad' registrado en el CNPQ. Dentro de cada uno de los proyectos se desarrollan investigaciones de iniciación científica, trabajos de 
finalización de curso de graduación y maestría en Diseño e investigaciones científicas de profesores. De esta manera, se realiza un seguimiento de las producciones y se busca medir sus resultados efectivos.

El proyecto AmaViva contempla los siguientes subgrupos productivos:

e) Aviva: Aviva Consciencia Colectiva, es un emprendimiento social que actúa en el segmento de moda ecológica y con destino de parte de las ganancias a proyectos sociales con niños en situación de vulnerabilidad.

f) EcoBanner: Desarrollo de bolsos y pequeños contenedores para automóvil, utilizando lonas de banners descartados.

g) Mútua: Producción de bolsos y carteras utilizando un rechazo de cuero y gemas.

h) RedCarpet: Desarrollo de vestidos de fiesta a partir de desechos de talleres de Joinville.

i) Tiwa: Producción de mochilas, estuches y neceseres utilizando telas de sombrillas y paraguas ya no utilizables.

De los grupos arriba citados pasaremos al análisis específico de uno de ellos: AVIVA Conciencia Colectiva. La intención aquí es evaluar las tecnologías en los materiales y procesos productivos de este subgrupo emprendiendo un análisis comparativo con el tema de la camiseta, anteriormente presentado.

AVIVA es una marca ambientalmente amigable y social, en fase de desarrollo. Nació a partir de la percepción de la necesidad de equidad en el ambiente laboral de la moda, de la preservación de los recursos naturales, de la posibilidad de generación de ingresos para personas en situación de vulnerabilidad y de la alternativa de destinación de beneficios para proyectos sociales con niños. Así, por medio de la moda y de los proyectos sociales, su intención es "avivar la conciencia colectiva y ambiental desde la infancia y formar una generación aún más crítica, que esté ligada a los verdaderos valores colaborativos". Se pretende, de forma práctica, actuar con transparencia de informaciones, divulgación de los impactos ambientales en los procesos, utilización de materiales menos agresivos al medio ambiente, cocción de los productos e inserción de la marca en la economía circular.

El actual sistema económico ha causado interferencias en la dinámica y estructuración familiar (material y emocional), ampliando las responsabilidades de la mujer en su sustento. Así, la referida marca social entiende que el impacto social primario ocurre en la generación de trabajo y renta, para las artesanas socias del proyecto de extensión, aliado a la capacitación (que incluye talleres para discutir sobre precio justo de mano de obra y formación de precio del producto final) y satisfacción profesional y personal de los agentes involucrados. El impacto secundario está orientado a inversiones en proyectos sociales con los niños. En su primera colección (en desarrollo), la planificación para el impacto ambiental está relacionada con la elección de materias primas recicladas y orgánicas (algodón orgánico, media malla y papel de algodón reciclado y poliéster reciclado de garaje pet), utilización de residuos en la construcción de las plantas ropa y artesanías y cuidados en el proceso, para reducir al mínimo posible la producción de basura. La clasificación de los beneficios sociales producidos por el negocio se basará en los indicadores propuestos por el IRIS (Impact Reporting and Investment Standards) (2016), que toma en consideración dos variables: los impactados con el negocio (quienes reciben los beneficios) y el beneficio social generado mejora en el aspecto social y medioambiental) (BACK et al., 2013).

Las buenas intenciones de AVIVA puntuan un anclaje teórico en principios de la sostenibilidad. Sin embargo, en la práctica, y eso es lo que nos interesa en esta reflexión, se hace necesario, como se ha hecho más arriba, en el caso de la camiseta, además de los procesos tecnológicos, considerar aspectos integrantes de los materiales y procesos productivos, así como la relación que se establece entre los agentes implicados. Así, con el propósito de ir adelante de las constataciones acerca del proyecto AVIVA, y considerando que se trata de un proyecto embrión, aún en fase de formato, se presenta en la tabla 1, con base en toda fundamentación apresurada en este artículo, y a partir de datos levantados en investigación de maestría (por Silvana Witkoski; orientación de Elenir Morgenstern, 2018), directrices, en formato tabla con una clasificación de tejidos en cuanto a su constitución. La intención es que el proyecto, además de los ideales conceptuales, efectúe en la práctica opciones de tejido que en su fabricación consideren el medio ambiente.

\begin{tabular}{|c|c|c|c|c|}
\hline & $\begin{array}{l}\text { Algodón } \\
\text { convencional }\end{array}$ & $\begin{array}{l}\text { Algodón } \\
\text { orgánico }\end{array}$ & Poliéster & Viscosa \\
\hline & Fibra natural & Fibra natural & $\begin{array}{l}\text { Fibra quími- } \\
\text { ca sintética }\end{array}$ & $\begin{array}{l}\text { Fibra quími- } \\
\text { ca artificial }\end{array}$ \\
\hline 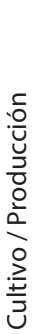 & $\begin{array}{l}\text { Monocultura } \\
\text { - causando } \\
\text { desequilibrio } \\
\text { en el suelo: } \\
\text { estéril, seco, } \\
\text { sin humedad, } \\
\text { erosión, } \\
\text { disminución } \\
\text { de materia } \\
\text { orgánica. }\end{array}$ & $\begin{array}{l}\text { Rotación } \\
\text { de cultivos }\end{array}$ & $\begin{array}{l}\text { Derivada de } \\
\text { combus- } \\
\text { tible fósil } \\
\text { natural no } \\
\text { renovable }\end{array}$ & $\begin{array}{l}\text { Gran parte } \\
\text { derivada } \\
\text { de árboles } \\
\text { de bosques } \\
\text { nativos (de- } \\
\text { forestación) }\end{array}$ \\
\hline
\end{tabular}




\begin{tabular}{|c|c|c|c|c|}
\hline & $\begin{array}{l}\text { Uso de } \\
\text { fertilizantes } \\
\text { químicos }\end{array}$ & $\begin{array}{l}\text { Compuesto } \\
\text { y Abono } \\
\text { orgánico }\end{array}$ & $\begin{array}{l}\text { Utiliza } \\
\text { grandes can- } \\
\text { tidades de } \\
\text { agua para el } \\
\text { enfriamiento }\end{array}$ & $\begin{array}{l}\text { La atmósfera } \\
\text { del cableado } \\
\text { es bastante } \\
\text { tóxica }\end{array}$ \\
\hline & $\begin{array}{l}\text { Uso de } \\
\text { pesticidas } \\
\text { químicos }\end{array}$ & $\begin{array}{l}\text { Trampas } \\
\text { para control } \\
\text { de plagas; } \\
\text { uso de insec- } \\
\text { tos benéficos }\end{array}$ & $\begin{array}{l}\text { Uso de } \\
\text { químicos no- } \\
\text { civos, como } \\
\text { lubricantes }\end{array}$ & - \\
\hline & $\begin{array}{l}\text { Uso de má- } \\
\text { quinas (com- } \\
\text { bustible) para } \\
\text { la cosecha, } \\
\text { desmotado, } \\
\text { pulverización }\end{array}$ & $\begin{array}{l}\text { Cosecha ma- } \\
\text { nual; capina } \\
\text { manual }\end{array}$ & $\begin{array}{l}\text { Utiliza gran } \\
\text { cantidad de } \\
\text { energía en } \\
\text { el proceso }\end{array}$ & - \\
\hline & $\begin{array}{l}\text { Uso de } \\
\text { colorantes } \\
\text { (teñido y } \\
\text { estampado) }\end{array}$ & $\begin{array}{l}\text { Colorantes } \\
\text { de bajo } \\
\text { impacto }\end{array}$ & $\begin{array}{l}\text { Uso de } \\
\text { colorantes } \\
\text { (teñido y } \\
\text { estampado) }\end{array}$ & $\begin{array}{l}\text { Uso de } \\
\text { colorantes } \\
\text { (teñido y } \\
\text { estampado) }\end{array}$ \\
\hline & $\begin{array}{l}\text { Uso excesivo } \\
\text { de agua (en } \\
\text { el proceso } \\
\text { y limpieza) }\end{array}$ & $\begin{array}{l}\text { Uso reducido } \\
\text { de agua }\end{array}$ & $\begin{array}{l}\text { Uso excesivo } \\
\text { de agua (en } \\
\text { el proceso } \\
\text { y limpieza) }\end{array}$ & $\begin{array}{l}\text { Uso excesivo } \\
\text { de agua (en } \\
\text { el proceso } \\
\text { y limpieza) }\end{array}$ \\
\hline & $\begin{array}{l}\text { Productos } \\
\text { químicos para } \\
\text { blanqueary } \\
\text { blanquear }\end{array}$ & $\begin{array}{l}\text { Sin apun- } \\
\text { tamiento }\end{array}$ & $\begin{array}{l}\text { Productos } \\
\text { químicos para } \\
\text { blanqueary } \\
\text { blanquear }\end{array}$ & $\begin{array}{l}\text { Productos } \\
\text { químicos para } \\
\text { blanqueary } \\
\text { blanquear }\end{array}$ \\
\hline 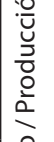 & $\begin{array}{l}\text { Procesos } \\
\text { demorados } \\
\text { y uso de } \\
\text { energía }\end{array}$ & $\begin{array}{l}\text { Uso reducido } \\
\text { de energía }\end{array}$ & $\begin{array}{l}\text { Procesos } \\
\text { demorados } \\
\text { y uso de } \\
\text { energía }\end{array}$ & $\begin{array}{l}\text { Procesos } \\
\text { demorados } \\
\text { y uso de } \\
\text { energia }\end{array}$ \\
\hline$\frac{\grave{ \pm}}{3}$ & $\begin{array}{l}\text { Fibras fuertes } \\
\text { y resistentes, } \\
\text { en menor } \\
\text { grado que } \\
\text { la sintética }\end{array}$ & $\begin{array}{l}\text { Fibras fuertes } \\
\text { y resistentes, } \\
\text { en menor } \\
\text { grado que } \\
\text { la sintética }\end{array}$ & $\begin{array}{l}\text { Fibras } \\
\text { fuertes y } \\
\text { resistentes, } \\
\text { generan pro- } \\
\text { ductividad }\end{array}$ & $\begin{array}{l}\text { Fibras fuertes } \\
\text { y resistentes, } \\
\text { en menor } \\
\text { grado que } \\
\text { la sintética }\end{array}$ \\
\hline & $\begin{array}{l}\text { Absorbe la } \\
\text { humedad }\end{array}$ & $\begin{array}{l}\text { Absorbe la } \\
\text { humedad }\end{array}$ & $\begin{array}{l}\text { Resistencia a } \\
\text { la humedad }\end{array}$ & $\begin{array}{l}\text { Absorbe la } \\
\text { humedad }\end{array}$ \\
\hline & $\begin{array}{l}\text { Se seca } \\
\text { rápidamente }\end{array}$ & $\begin{array}{l}\text { Se seca } \\
\text { rápidamente }\end{array}$ & $\begin{array}{l}\text { Se seca } \\
\text { rápidamente }\end{array}$ & $\begin{array}{l}\text { Se seca } \\
\text { rápidamente }\end{array}$ \\
\hline & $\begin{array}{l}\text { Desbota un } \\
\text { poco con } \\
\text { el tiempo }\end{array}$ & $\begin{array}{l}\text { Desbota un } \\
\text { poco con } \\
\text { el tiempo }\end{array}$ & $\begin{array}{l}\text { No se } \\
\text { desvanece }\end{array}$ & $\begin{array}{l}\text { No se } \\
\text { desvanece }\end{array}$ \\
\hline & $\begin{array}{l}\text { Amasa } \\
\text { levemente }\end{array}$ & $\begin{array}{l}\text { Amasa } \\
\text { levemente }\end{array}$ & No amasa & $\begin{array}{l}\text { Amasa y } \\
\text { encoge con } \\
\text { facilidad }\end{array}$ \\
\hline & Luz & Luz & Luz & Luz \\
\hline & $\begin{array}{l}\text { No hay } \\
\text { bolitas }\end{array}$ & $\begin{array}{l}\text { No hay } \\
\text { bolitas }\end{array}$ & $\begin{array}{l}\text { Produce boli- } \\
\text { tas en fricción }\end{array}$ & $\begin{array}{l}\text { No hay } \\
\text { bolitas }\end{array}$ \\
\hline & $\begin{array}{l}\text { No retiene } \\
\text { el calor, la } \\
\text { piel respira }\end{array}$ & $\begin{array}{l}\text { No retiene } \\
\text { el calor, la } \\
\text { piel respira }\end{array}$ & $\begin{array}{l}\text { Reten el calor } \\
\text { del cuerpo, } \\
\text { generando } \\
\text { sudory } \\
\text { mal olor }\end{array}$ & $\begin{array}{l}\text { No retiene } \\
\text { el calor, la } \\
\text { piel respira }\end{array}$ \\
\hline & $\begin{array}{l}\text { Biodegra- } \\
\text { dable }\end{array}$ & $\begin{array}{l}\text { Biodegra- } \\
\text { dable }\end{array}$ & $\begin{array}{l}\text { No biode- } \\
\text { gradable } \\
-400 \text { años } \\
\text { para des- } \\
\text { componerse }\end{array}$ & $\begin{array}{l}\text { Biodegra- } \\
\text { dable }\end{array}$ \\
\hline
\end{tabular}

Tabla 01 - Materia prima para la confección de la camiseta. Fuente: Autores.
La tabla, desarrollada a partir de investigación bibliográfica y de campo (investigación de maestría en desarrollo, por Witkoski; Morgenstern, 2018) contiene informaciones acerca de las materias primas más utilizadas en la industria textil y moda: algodón, poliéster y viscosa (COLERATO, 2017; Dalila Têxtil, 2017; Fibracel, 2000; Hskins te, 2014; jones, 2005). Ella compara los diferentes productos, desde su producción o cultivo, en el caso del algodón, abriéndose en convencional y orgánico, hasta el tejido listo; establece características de cada materia prima, procesos de acabado y situación después de la eliminación; destaca datos que evidencian calidad y cuidado con el medio ambiente. Se entiende que los resultados presentados en la tabla anterior pueden contribuir con emprendimientos sociales de moda, como es el caso de la aviva, orientando sobre opciones conscientes y sostenibles.

\section{MODA Y TECNOLOGÍA EN CONTEX- TO EUROPEO: PRACTICAS SITUADAS EN BARCELONA}

La historia de la extensión universitaria es reciente en el contexto de la universidad. En una investigación anterior (HERMES; MORGENSTERN, 2017), constatamos que la extensión universitaria brasileña está configurada en moldes diferentes de los europeos.

El área de extensión universitaria se configura en uno de los tres pilares que estructuran la universidad: enseñanza, investigación y extensión. Su origen proviene de Europa, en el siglo pasado, y su objetivo principal era difundir la enseñanza técnica producida por la universidad. Partió de Inglaterra la proposición de que las instituciones de enseñanza deberían colaborar con la sociedad, en el sentido de contribuir por medio de conocimiento producido. La extensión estadounidense, por otro lado, desde su llegada, mantuvo la función de prestación de servicios. La extensión en américa latina se desarrolló en movimientos sociales, con la premisa de que la cultura fuese diseminada para las clases populares (MELO NETO, 2002).

Establecer un paralelo entre prácticas de extensión universitaria (inclinadas a la producción de moda y tecnología) en Brasil y en Barcelona, es inviable, considerando la naturaleza de las prácticas en ambos países. La extensión universitaria en Barcelona está dirigida a reforzar los programas de estudio que difícilmente observan los cambios sociales en el territorio del diseño. Los proyectos sociales, orientados a las producciones de moda, representan una parte casi imperceptible en las prácticas de la ciudad. De hecho, sí que existen proyectos que revisan de forma crítica esta problemática, pero es más fácil encontrarlos en el activismo 
ciudadano o en los talleres urbanos. El colectivo "makea tu vida" con sede en Barcelona lleva tiempo explorando la potencia del "diseño abierto" entendido como un diseño más responsable. Su relato versa así: "makea tu vida es una entidad sin ánimo de lucro de carácter social y educativo, que trabaja en el territorio que existe entre los mundos del diseño y la ecología" (http://www.makeatuvida.net/). Los trabajos de este colectivo son solo un ejemplo de los avances en el mundo activista del diseño y que reflejan lo anquilosado de la extensión universitaria en el sur de Europa.

Otro factor que, posiblemente, intimida la formación de grupos de producción en moda, volcados a la comunidad y al medio ambiente, es la impactante producción de moda industrial en España, y ahí nos referimos, por ejemplo, a las empresas de fast fashion como zara y mango. Los precios son muy bajos, en comparación con el comercio de productos de moda en Brasil, este factor facilita el consumo que abastece al mercado local y también al internacional (a precios más elevados). Estas empresas de comercio global de moda, son famosas por no incluir en su adn corporativo los aspectos sociales o ambientales que afectan a los países en donde intervienen con sus productos. Según varias críticas, por investigadores sociales y activistas ambientales, estas empresas promueven sus productos a través de la amplia diseminación de puntos de venta y facilitación del comercio a través de precios muy bajos, que sólo son posibilitados por la falta de inversión en tecnologías que tomen en cuenta el respeto por el medio ambiente.

Consideramos interesante presentar, en esta parte final, un comparativo entre las prácticas productivas, que congregan moda y tecnología, situadas en dos contextos diversos, Brasil (Santa Catarina) y España (Barcelona). Este comparativo fue debido a la observación del contraste existente entre las prácticas en los dos contextos histórico- geográficos observadas a lo largo de tres meses de etapa postdoctoral desarrollada por esta investigadora brasileña (que os escribe) en Barcelona, bajo la supervisión del también autor del presente artículo, profesor Efraín Foglia.

\section{CONSIDERACIONES FINALES}

El artículo, resultado de estudios y reflexiones realizadas durante el posdoctorado (por Elenir Morgenstern bajo la supervisión del Prof. Dr. Efrain Foglia) en la Universidad de Barcelona (UB), abordó cuestiones relacionadas con las fronteras del diseño. Se enfocó en las prácticas relacionadas con los campos del diseño de la moda y la tecnología para el desarrollo de productos. En este sentido, el artículo destaco teorías conexas a una producción de diseño de moda sostenible evidenciando prácticas relacionadas con proyectos sociales, de extensión universitaria, que invierten en tecnología social que, por medio de manufacturas, en sus métodos, procesos y materialidad, minimicen impactos ambientales.

A partir del cruce entre los enfoques teóricos que anuncian tecnologías de última generación, aplicables en el desarrollo de artefactos de moda, y las prácticas analizadas junto a los proyectos sociales, de extensión universitaria, impulsados por los saberes del diseño de moda, en el contexto brasileño, el artículo planteo cuestionamientos acerca de la efectiva contribución de los avances tecnológicos al medio ambiente, específicamente en el campo de la moda.

La reflexión presentada, con el fin de transponerse a la visión romántica acerca del potencial de las tecnologías para el campo de la moda, indago de hecho, y en qué medida, las tecnologías sociales y los procesos tecnológicos aplicados en el desarrollo de artefactos de moda pueden contribuir con la sostenibilidad, minimizando los impactos ambientales. Esta tentativa, en su conjunto, discurre acerca del diseño de moda y perspectivas sostenibles; describió tecnologías actualmente aplicables a los artefactos de moda; ha presentado un contexto ilustrativo escudriñando la comprensión teórica por medio de un breve historial sobre la evolución tecnológica de la pieza "camiseta"; contextualizó prácticas de tecnología social promovidas por proyectos de extensión universitaria en Brasil; y, finalmente, analizó y emitió posicionamientos de valor por medio de cruce entre las reflexiones teóricas y los casos prácticos en contexto brasileño y español.

Al final de las reflexiones, estamos convencidos de que la aplicación de la tecnología, al contrario de lo esperado, puede generar nuevos problemas al medio ambiente $y$, por consiguiente, a las sociedades. Pensamos que los intercambios comerciales deben ser consecuencia de un proceso de reflexión en sintonía con el momento actual de nuestra sociedad, entendiendo que ese debate, debe ser promovido en el escenario de una sociedad que pretende ser participativa y cuestionadora de los sistemas provenientes del capitalismo voraz. Creemos que la evolución tecnológica no puede basarse en la referencia de los beneficios de vender tecnologías, si en lo que se hace necesario averiguar concretamente cuáles son los beneficios sociales que obtenemos al utilizarla.

\section{REFERENCIAS}

AVELAR S. Moda, globalização e novas tecnologias. Estação das Letras, São Paulo, 2009. 
BACK, Nelson; OGLIARI, André; DIAS, Acires; SILVA, Jonny Carlos da. Projeto Integrado de Produtos: Planejamento, Concepção e Modelagem. Barueri: Manole, 2013.

BERGMANN M., MAGALHÃES C. Materiais híbridos: natureza têxtil em transformação por meio da eletrônica e da nanotecnologia. In: CONGRESSO INTERNACTIONAL E WORKSHOP DESIGN \& MATERIAIS, 2017. Anais... Campinas, Galoá, 2018. Disponible en: $<$ https://proceedings.science/dm/trabalhos/materiais-hibridos-natureza-textil-em-transformacao-por-meio-da-eletronica-e-da-nanotecnologia>. Acceso en el: 15 out. 2018.

BERLIM L. Moda e sustentabilidade: uma reflexão necessária. Estação das Letras, São Paulo, 2012.

BEZERRAFC.Oqueétecido poliéster? eCycle. Disponible en: <https://www.ecycle.com.br/component/content/ article/73-vestuario/2900-poliester-fibra-popular-reciclavel-riscos-ambientais-pet-politereftalato-de-etileno-tecidos-malhas-roupas-filmes-filtros-tintas-pneus-led-historia-petroleo-nao-biodegradavel-reciclavel-mistura-danos-ambienta-microplasticos-alternativas-organicos.html>. Accesso en el: 27 set. 2017. BOUCHER F. História do vestuário no ocidente: Da origem aos nossos dias. Cosac Naify, São Paulo, 2010.

BOURDIEU P. O costureiro e sua grife: contribuição para uma teoria da magia. In: A produção da crença: contribuição para uma economia dos bens simbólicos. 3. ed. Zouk, Porto Alegre, 2006.

BROWNE MA et al. Accumulation of microplastic on shorelines worldwide: sources and sinks. Environmental Science \& Technology, 45:21, pp 91759179, 2011. Disponible en: <http://pubs.acs.org/doi/ ipdf/10.1021/es201811s>. Accesso en el: 27 set. 2017.

CANCLINI NG. Culturas híbridas: estratégias para entrar e sair da modernidade. Edusp, São Paulo, 2003.

CAPRA F. As conexões ocultas: ciências para uma vida sustentável. 13. ed. Cultrix, São Paulo, 2013.

COLERATO M. 4 razões para você pensar duas vezes antes de optar pela viscose. Modefica, 2017. Disponible en: <http://www.modefica.com.br/4-razoes-viscose-roupa-insustentavel/\#.Wdvfh_ISzIU>. Accesso en el: 9 out. 2017.

DALILA TÊXTIL. Disponible en: <http://www.dalilatextil.com.br>. Accesso en el: 27 set. 2017.

FIBRACEL. Fibra cortada de viscose: Manual Técnico para Fiações. 2000. Disponible en: <http://www.abqct.com. br/artigost/Manual\%20Fia\%C3\%A7\%C3\%A30\%20 Viscose.pdf>. Accesso en el: 10 out. 2017.
FLETCHER K, GROSE L. Moda \& Sustentabilidade: design para mudança. Senac, São Paulo, 2011.

FOGLIA E. Cambio. IN Desconstruyendo el Manifiesto Marker. Edición Trànsit Projectes/MakerConvent, 2017. FORTY A. Objetos de desejo: Design e sociedade desde 1750. Cosac Naify, São Paulo, 2007.

HERMES L, MORGENSTERN E. Design e projetos sociais: processos e efeitos da extensão universitária. Relatório Técnico. Joinville, 2017. Disponible en: <www.univille.edu.br/account/ppgdesign/VirtualDisk.html/.../34. HERMES_Leticia.pdf $>$. Acceso en el: 25 set. 2017.

HSKINS TE. Stitched Up - The Anti-Capitalist Book of Fashion. Pluto Press, Londres, 2014.

JONES SJ. Fashion design: manual do estilista. Cosac Naify, São Paulo, 2005.

MANICHINELLI M. Desconstruyendo y rehaciendo las identidades de los Makers. IN Desconstruyendo el Manifiesto Marker. Edición Trànsit Projectes/ MakerConvent, 2017.

MELO NETO JF de. Extensão universitária: bases ontológicas. Extensão Universitária: Diálogos Populares, 13, 2002.

RHODIA SOLVAY GROUP. Cases de sucesso: Emana. Disponible en: <http://www.rhodia.com.br/pt/company/inovacao/cases-de-sucesso/Success-StoryEmana.html>. Accesso en el: 25 set. 2017.

RIKEN. A solar cell you can put in the wash. 2017. Disponible en: <http://www.riken.jp/en/pr/ press/2017/20170919_2/>. Accesso en el: 25 set. 2017. SASAWASHI. About Sasawashi. Disponible en: <http:// sasawashi.com/en/about/>. Accesso en el: 24 set. 2017. SCHULTE NK, LOPES L. Sustentabilidade ambiental: um desafio para a moda. Modapalavra e-periodico, 1:2, pp 30-42, ago-dez. 2008. Disponible en: <http://www. periodicos.udesc.br/index.php/modapalavra/article/ view/7601/5107>. Accesso en el: 10 out. 2017.

SETE SSK, MORGENSTERN E. Fibras Naturais na produção de fios têxteis: melhoramentos no estudo do uso da fibra de Paineira e aplicações em artefatos de Design de Moda. In: CONGRESSO INTERNACIONAL DESIGN E MATERIAIS, 2., 2017. Anais... 2017.

SOUZA MCM de. A produção de têxteis de algodão orgânico: uma análise comparativa entre o subsistema orgânico e o sistema agroindustrial convencional. Agric., São Paulo, 2000.

THE GUARDIAN. Spinning sour milk into silky fibres. The Guardian. Disponible en: <https://www.theguardian.com/sustainable-business/sour-milk-fibres-textiles-qmilk>. Accesso en el: 25 set. 2017. 
VEZZOLI C. Cenário de design para moda sustentável. In: PIRES BD (Org.). Design de moda: olhares diversos. Estação das Letras, São Paulo, 2008.

\section{AUTORES}

https://orcid.org/0000-0002-6384-6068

ELENIR CARMEN MORGENSTERN, Dra. | Univille - Mestrado Profissional em Design e Graduacao em Design | Cidade: Joinville, SC, Brasil | Correspondência para: R. Estrada da Ilha, 2419 Joinville; CEP.: 89239250 | email: ele.stern18@ gmail.com

ORCID: 0000-0002-5144-9161

EFRAÍN FOGLIA, Dr.| Profesor e Investigador de la Universitat Oberta de Catalunya, UOC. | Correspondência para: Pintor Fortuny 17-19, 20 4a, 08001. Barcelona, España. | E-maill: efogliar@uoc.edu

ORCID: 0000-0001-6608-6853

SILVANA SILVA REITER WITKOSKI, M.Sc. | Mestrado em design profissional | Correspondência para: R. Bernardo Dornbusch, 2306- Vila Lalau, Jaraguá do Sul, SC, 89.256-214 | E-maill: vanawitkoski@gmail.com

ORCID: https://orcid.org/0000-0002-0889-8770

LETICIA HERMES, M.Sc. | Universidade da Região de Joinville - UNIVILLE | Mestrado Profissional em Design | Joinville, SC - Brasil | Correspondência para: R. Benedito Osvaldo Lecques, 100. Apto 153-A - Jardim Aquarius, São José dos Campos - SP, 12246021 | E-maill: lethermes@gmail.com

https://orcid.org/0000-0002-4502-1767

HELENA MORGENSTERN ZAMBERLAN | Universidade da Região de Joinville - UNIVILLE | Correspondência para: Rua Estrada da Ilha, 2419 - Pirabeiraba, Joinville- SC, 89.239-250, Brasil | email: hmzamberlan@gmail.com

\section{COMO CITAR ESTE ARTIGO}

MORGENSTERN, Elenir Carmen; FOGLIA, Efraín; WITKOSKI, Silvana Silva Reiter; HERMES, Letícia; ZAMBERLAN, Helena Morgenstern. Las Fronteras Entre Diseño De Moda Y Tecnología: Posibilidades Para Una Producción Sostenible. MIX Sustentável, [S.I.], v. 6, n. 4, p. 145-158, ago. 2020. ISSN 24473073. Disponível em:<http://www.nexos.ufsc.br/index.php/ mixsustentavel>. Acesso em: dia mês. ano. doi:https:// doi.org/10.29183/2447-3073.MIX2020.v6.n4.145-158.

DATA DE ENVIO: 27/05/2020

DATA DE ACEITE: 31/07/2020 
